\title{
In vivo personalized finite element modeling of the knee joint derived from MRI images
}

\author{
Methodology applied on one contact problem \\ including bones, cartilages and menisci
}

\author{
Morgan Sangeux* — Frédéric Marin* — Fabrice Charleux** \\ Marie-Christine Ho Ba Tho* \\ * Laboratoire de biomécanique et génie biomédical \\ CNRS-UTC 6600 \\ BP 20529, F-60205 Compiègne cedex \\ morgan.sangeux@gmail.com,\{frederic.marin,christine.hobatho\}@utc.fr \\ ** Centre d'Imagerie Medicale Avancée \\ Compiègne, France \\ fabrice.charleux@wanadoo.fr
}

ABSTRACT. This paper adresses the methodology used to model the knee joint in vivo from MRI images. The knee joint model obtained is subject specific. The paper presents all the treatment procedure: Geometrical acquisitions, Joint movement analysis, Meshing techniques and nonlinear finite element modeling with contact between the bones, the cartilage and the menisci. The model provides the contact pressure applied on the various components of the joint for one normal subject.

RÉSUMÉ. Cet article présente la méthodologie de modélisation personnalisée in vivo de l'articulation du genou à partir d'images IRM. Les étapes de traitement présentées sont : acquisition de la géométrie des structures, calcul du mouvement de l'articulation, construction du maillage des structures et calculs éléments finis non linéaires incluant le contact entre les os, le cartilage et les ménisques. Le modèle ainsi obtenu permet de déterminer les efforts de contact appliqués aux différentes structures de l'articulation du genou sain.

KEYWORDS: knee joint, MRI, contact modelling.

MOTS-CLÉS : articulation du genou, imagerie médicale IRM, modélisation du contact.

DOI:10.3166/EJCM.18.81-92 @ 2009 Lavoisier, Paris

EJCM - 18/2009. Numerical models in biomechanics, pages 81 to 92 


\section{Introduction}

Finite element modeling has expanded in the biomechanical field the past three decades. This tool is generally used to study prosthesis adaptation to their mechanical environnement and to evaluate different surgery strategies or replacement materials. In the case of the knee, several studies were focused on the total knee prosthesis, the crucciate or lateral ligaments reconstruction or replacement and the menisci surgeries. In most of the models, the geometrical informations of the different structures comes from cadaveric pieces and are obtained with X-ray based acquisitions (fluoroscope, scanner) coupled with MRI or alone. According to our knowledge, few studies built a complete knee in vivo model.

Although finite element modeling is generally used as a prediction tool one can imagine that it can also be used as a diagnostic or comprehension tool. Indeed a mechanical model of the joint can provide substantial new informations compared with traditional ways. But dealing with biomechanics, personalization of the models proposed are of great interest since small changes in geometry, loads conditions, material properties may produce significant different results or conclusion. The purpose of the present paper is then to present the methodology used to address this objective.

\section{Materials and methods}

Each step of the methodology employed in this study starts with a specific MRI acquisition procedure. All the MRI acquisitions were performed with the 1.5T Signa MRI scanner (GE Medical Systems, Milwaukee, WI, USA) with a radiologist specialized in osteoarticular joints. The modeling software employed in this study were:

- image processing : SIPCINSERM (Ho Ba Tho, 1993) and Amira (Mercury Computer Systems Inc, Merignac, France);

- geometrical modeling : PATRAN (MSC.Software, California, USA);

- finite element solver : MARC (MSC.Software, California, USA).

The methodology used to build the finite element model can be split into two parts:

- acquisition of the kinematic data; These data were used as boundary conditions for the finite element model;

- finite element modeling procedure.

\subsection{Kinematics data}

\subsubsection{Data collection}

Measurements were made on the right knee of one healthy volunteer (male, 27 years old). The volunteer underwent a clinical knee exam performed by an orthopedic surgeon at the Polyclinique St Côme in Compiègne and signed in an informed consent. 
During the measurement the subject lay down on the MRI table in supine position. His ankle was fixed to a custom-made foot drive device (Figure 1 ) manufactured with MR compatible materials. The foot drive device was designed in order to allow a reproducible active knee movement within the MRI tunnel. The foot drive device was composed of two parts. The first part was a plastic rail fixed onto the MRI table. The second part was composed of a rigid ankle orthosis (modified Aircast,Summit,USA) attached to the subject's foot and driven by the subject. Subject knee extension was performed against the constraint of two elastic bands linking the sliding part which bore subject's foot to the fixed rail.

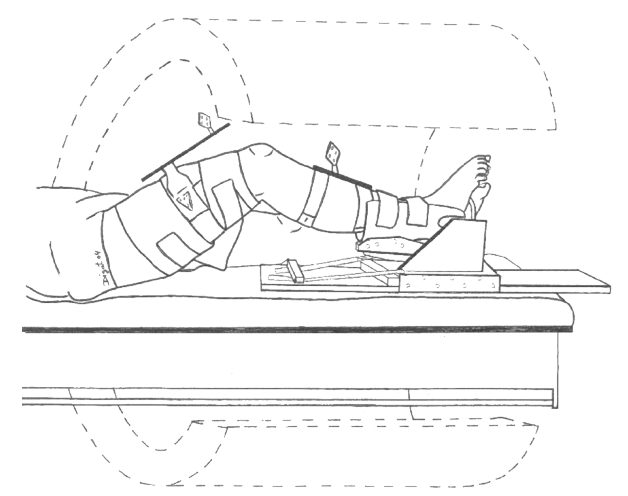

Figure 1. The foot drive device allow a repeatable and active knee movement. The elastic bands enable the knee joint loading

Data from the 3D volume of the knee and the external marker sets were acquired while the subject performed a sequential loaded knee extension within the MRI scanner.

The acquisition parameters choice came from a compromise between the total examination time and the spatial resolution. In comparison with standard MRI knee protocol a higher field of view is necessary in order to take into account the movement of the knee. Furthermore, added slices are required to obtain a volume acquisition in order to perform a 3D model. Acquisition parameters are described in Table 1.

The position of the subject was adjusted as soon as his patella touched the top of the MRI tunnel at approximately $90^{\circ}$ of knee flexion. From this position the subject performed a knee extension. During the knee extension the subject was asked to pause three times until full extension. A set of notches placed on the foot drive device rail allowed the subject to track each position. This procedure was chosen in order to get the maximum range of flexion. During each pause the volume defined by the joint's bones was acquired with sagittal images of a routine MRI sequence. The total acquisition time per subject was 10 to 15 minutes, this time included subject installation and all acquisition for four positions. 
Prior to this procedure and outside the MRI room a piezoelectric force sensor was placed between the heel of the subject and the sliding part so that it records the reaction force under the subject's heel in the direction of the subject's tibia. This reaction force can therefore be viewed as a compression force in the knee (from tibia to the femur) during the active loaded knee extension movement.

\subsubsection{Kinematics computation procedure}

Sagittal images were then imported into the image processing software SIP. There, the femur and tibia outlines for each of the four positions were detected semiautomatically and described with spline curves. SIP first thresholds the MRI images, then the examiner just needs to select a point on the image and from this point or a small area around this point the software track and follow the threshold border to determine a closed outline. Then these outlines were imported into the pre-post finite element software PATRAN (Ho Ba Tho, 2003). Solid models of the bones were processed from the outlines as follows.

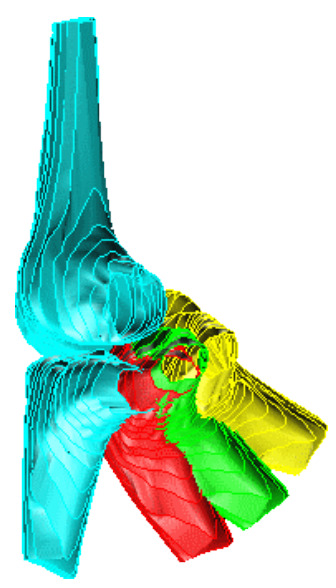

Figure 2. Relative movement of the tibia after registration of all femur models

1) Solid models of the tibia and femur were built from PATRAN geometrical functions. In particular the outlines spline curves were interpolated to create a surface that describe the bones.

2) A region of interest was automatically selected so that the total length along the long axis of the femurs and the tibia models was $150 \mathrm{~mm}$ and $100 \mathrm{~mm}$ respectively.

3) A coordinate frame based on the inertia axes of the bones models was computed and attached to each bone.

4) The femur and tibia of the extension acquisition were chosen as the reference and registered on their flexion corresponding bones. A first registration guess was achieved by the superimposition of the inertia axes of the bones, then, the Trimmed 
Iterative Closest Point (ICP) (Chetverikov et al., 2005) was processed on the models point clouds to adjust the registration of the bones.

5) All the femur models were superimposed in order to obtain a single femur with 4 tibia in the different finite positions.

6) The tibia movement with respect to the femur (Figure 2) was described with helical axes.

This study was a part of (Sangeux et al., 2006) from which the movement of 10 other volunteers was recorded in order to determine the relative movement of external marker set relative to their underlying bones.

\subsection{Finite element modeling}

\subsubsection{Data collection and image processing procedure}

An anatomical MRI acquisition was performed on the right knee of the same volunteer. The acquisition was performed in a static position and the MRI knee coil were used for image quality purpose. Different MRI sequences were tested in order to choose the one which gives a good contrast for all the structure of interest. An example of the various sequences used is shown on Figure 3

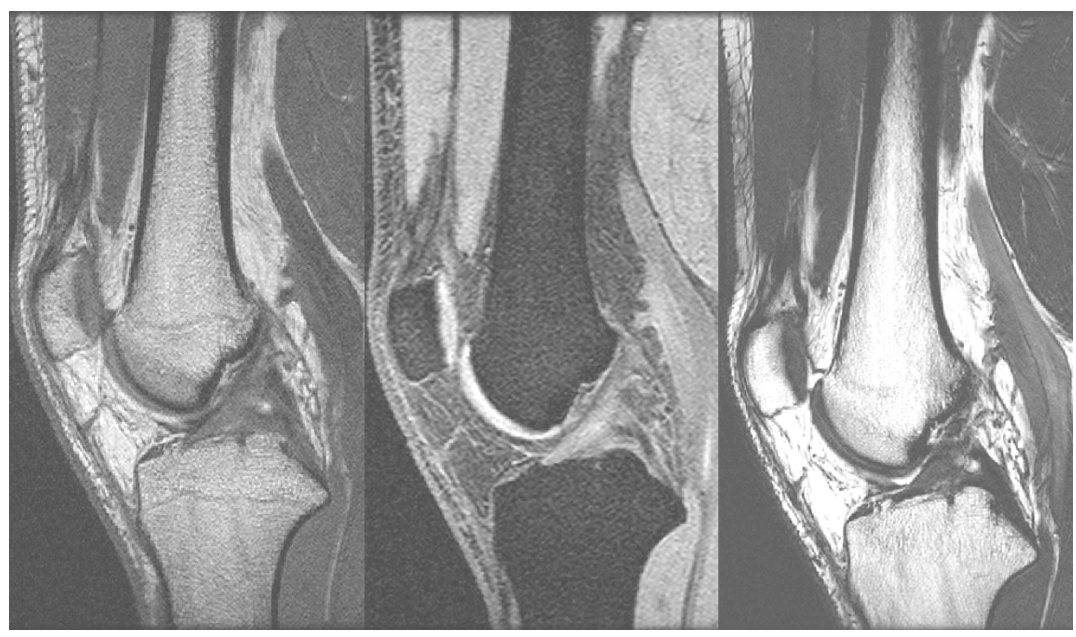

Figure 3. The different MRI sequences tested, from left to right: $3 D T 1,3 D T 2$ fat suppression and 3 D FIESTA

From Figure 3 the one chosen was the 3D FIESTA, this choice will be further discussed in the results section. Selected parameters for the sagittal images acquisition are described in Table 1 
Table 1. MRI acquisition parameters

\begin{tabular}{lcc}
\hline & Kinematics acquisition & Finite element modeling acquisition \\
\cline { 2 - 3 } Flip Angle & $50^{\circ}$ & $65^{\circ}$ \\
Repetition Time & $2.914 \mathrm{~ms}$ & $6.184 \mathrm{~ms}$ \\
Echo time & $1.096 \mathrm{~ms}$ & $1.948 \mathrm{~ms}$ \\
\hline Coils used & Body & Quad Knee \\
\hline Field of View & $460 \times 460 \mathrm{~mm}$ & $180 \times 180 \mathrm{~mm}$ \\
Image Matrix & $512 \times 512 \mathrm{pixels}$ & $512 \times 512$ pixels \\
Slice thickness & $3 \mathrm{~mm}$ & $2 \mathrm{~mm}$ \\
Interscan spacing & $0 \mathrm{~mm}$ & $-1 \mathrm{~mm}$ \\
\hline Voxel size & $0.89 \times 0.89 \times 3 \mathrm{~mm}$ & $0.35 \times 0.35 \times 1 \mathrm{~mm}$ \\
\hline
\end{tabular}

The image data were then imported in a commercial image processing software (Amira, Mercury Computer Systems) and the outlines of the bones (Femur, Tibia), the cartilage layers and menisci were processed. The outlines were selected/corrected in the three anatomical planes (frontal, axial and sagittal) with Amira manual and semi-automated tools. The most used tools were the manual brush, the dynamic region growing and the active contours, blow tool. The outlines were then exported to PATRAN as piecewise linear curves.

\subsubsection{Finite element model}

To find a general methodology for this step was a real challenge dealing with personalized anatomical structures. The following modeling procedure was found to give the best quality solid mesh without simplifying anatomical geometry. The mesh quality criteria were the aspect ratio of the elements in a particular structure, the twist of the elements and the node distribution homogeneity.

The idea was to build an initial surface mesh, then compute the vector field of the structure of interest thickness and finally swept the surface elements into solid elements. This procedure was applied to the cartilage layers and menisci. The outlines of these structures were split into 2 parts, the internal (closest to the bones) and the external, both parts modeled with interpolated spline curves from the imported data curves/points. From the internal and external curves two BSpline surfaces were built.

- The internal surface was meshed with $1.5 \mathrm{~mm}$ edge length quad elements, this element size will be the size of the final mesh. The external surface was meshed with the smallest elements allowed, $0.5 \mathrm{~mm}$, in order to get the most dense point cloud.

- A self running $\mathrm{C}$ function was used to compute the element based normal distance between internal and external meshes. A PCL (Patran Command Language) user-menu was build to launch the $\mathrm{C}$ function, import the results and convert them into a FEM dependent vector field,

- All the elements of the internal mesh were swept according to this vector field. 
All the meshing procedure steps are presented in Figure 4.

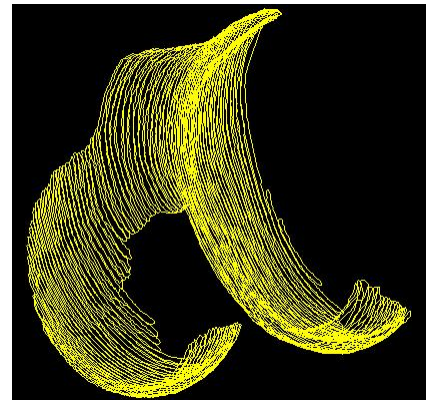

a)

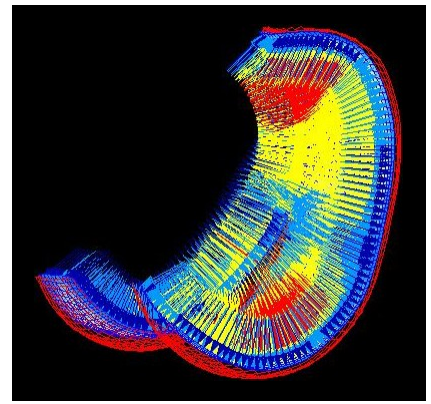

c)

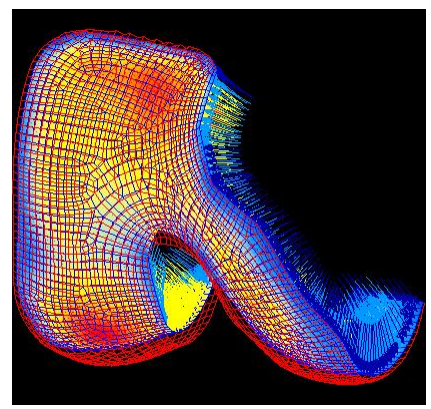

b)

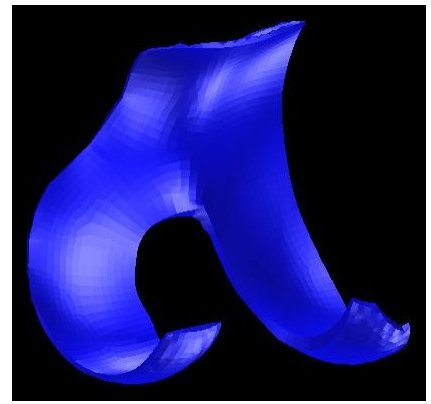

d)

Figure 4. The femoral cartilage layer building: a) Outlines import; b) and c) After division in internal and external surfaces and meshes, vector field computation is shown (color code: distance between the two meshes, range 0 to $4 \mathrm{~mm}$ ); d) Sweeping of the based mesh according to the vector field computed

The cartilage layers were meshed with unstructured hexahedral elements whereas menisci were meshed with structured hexahedral elements. Elements of the menisci were aligned in the circumferential direction for easier assignment of a local coordinate system to model transverse isotropy.

In order to determine the geometrical accuracy of the finite element model two tests were performed. The first test was to build the femoral cartilage layer by two different operators. The second one was to build the femoral cartilage layer by the same operator but from the different MRI acquisition sequences tested. The results were compared according to the cartilage layer thickness maps obtained from the different operators or MRI sequences.

\subsubsection{Load and boundary conditions}

Contact were defined between both femoral and tibial cartilage layers and these layers with the menisci. The contact were defined as frictionless as the cartilage to 
cartilage friction coeffcicient was estimated to [0.005-0.02] which is ten times less than an ice on ice at $0^{\circ} \mathrm{C}$ contact. A double-sided no penetration condition was applied.

To apply the bone kinematics, the femur and tibia were modeled as rigid surfaces, this hypothesis was found to be sufficiently accurate in the literature (Donahue et $a l ., 2002)$. The rigid surfaces were composed of quad elements from the internal parts of the cartilage layers. The tibial surface was controlled with force and the femur surface was controlled with velocity. The force record by the force sensor on the foot drive device was applied to the control node of the tibial rigid surface. This control node was allowed to move only in the direction of the force. The personalized kinematics was applied to the femur with the Marc user subroutine UMotion which prescribed for each increment the center of rotation, the rotation axis cosines and rotation amplitude around this axis of the rigid surface. Four steps were defined, first a tibial reaction force ramp up and then three rotations around the helical axes computed with the kinematics procedure.

Contact between these surfaces and the cartilage layers were modeled as glued so no relative displacement was allowed.

Finite element computations were performed with or without the menisci. When menisci were added to the model the most external nodes of the anterior and posterior horns of each meniscus were fixed.

\subsubsection{Material properties}

Material properties for the model were derived from the literature. In particular the Taguchi-based optimized parameters determined by (Yao et al., 2006) were used in this model. A wide range of properties can be found in the literature for articular cartilage, depending on loading rate and experimental conditions. In the conditions of the present experiment the cartilage was modeled as isotropic and linear elastic.

The menisci were modeled as linear elastic, transversely isotropic material (Donahue et al., 2003). Material properties of these two structures are reported in Table2.

Table 2. Material properties and elements type used for the cartilage and menisci

\begin{tabular}{llll} 
Structure & \multicolumn{2}{c}{ Mechanical properties } & Elements type \\
\hline \multirow{2}{*}{ Cartilage } & \multicolumn{2}{c}{$\mathrm{E}=5 \mathrm{MPa}$} & Solid \\
& \multicolumn{2}{c}{$\nu=0.45$} & Hybrid Herrman \\
\multirow{4}{*}{ Menisci } & $\mathrm{E}_{\theta}=125 \mathrm{MPa}$ & $\mathrm{E}_{r}=\mathrm{E}_{z}=27.5 \mathrm{MPa}$ & \\
& $\mathrm{G}_{\theta r}=\mathrm{G}_{\theta z}=2 \mathrm{MPa}$ & $\mathrm{G}_{r z}=10.34 \mathrm{MPa}$ & Solid \\
& $\nu_{\theta r}=\nu_{\theta z}=0.1$ & $\nu_{r z}=0.33$ & Standard formulation \\
\hline
\end{tabular}

\subsubsection{Qualitative control of the finite element results}

A finite element model based on in vivo measurements is difficult to validate as no information on the internal loading conditions are known. In this case the finite ele- 
ment model was controlled with the comparison between the predicted and effective contact area in the extension position.

Indeed from MRI images the contact surface area was determined by a nodal distance computation. The nodal distance function previously described was used. This function gave us the distance map between the external parts of the cartilage layers of the femur and the tibia.

\section{Results}

\subsection{Geometrical accuracy of the finite element model}

Figure 5 shows the results obtained when the same MRI images are treated by two different operators.

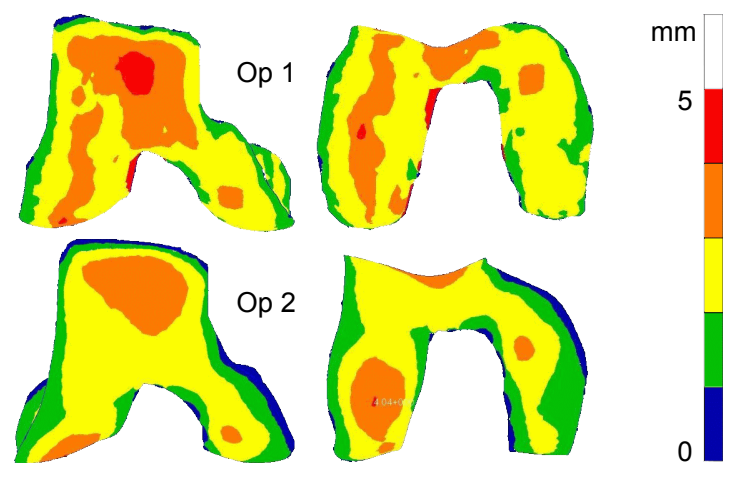

Figure 5. Femoral cartilage layer thickness map in mm for operator 1 and 2

Figure 6 6 shows the results obtained when the same operator treats the same femoral cartilage layer from various MRI acquisition sequences.

These results show that the expected accuracy is about $1 \mathrm{~mm}$ on the cartilage thickness. It shows also that the variations observed with different MRI sequences are greater than the one observed from different operators. Finally the MRI sequence chosen, 3D FIESTA, presents average results between the two other ones.

\subsection{Finite element model results}

Figure 7 presents the control test of the finite element computation in the extension position. It can be seen that the contact results (on the left) are located in the same position than the expected contact area seen on MRI images from nodal distance.

A complete simulation was performed for the model where menisci were removed. The contact area and the contact pressure on the cartilage layers during the knee flex- 


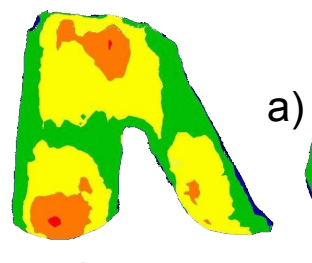

a)
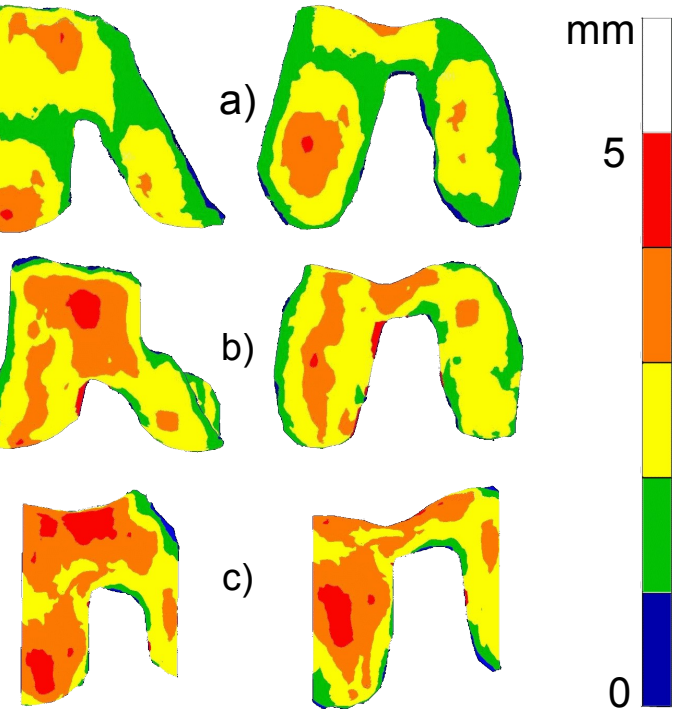

c)

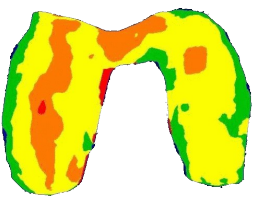

)

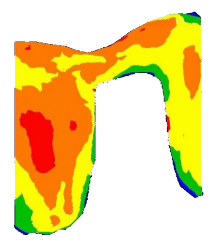

0

Figure 6. Femoral cartilage layer thickness map in $\mathrm{mm}$ from operator 1 for the 3 different MRI sequences, a) 3D T1 b) 3D FIESTA c) 3D T2 fat suppression

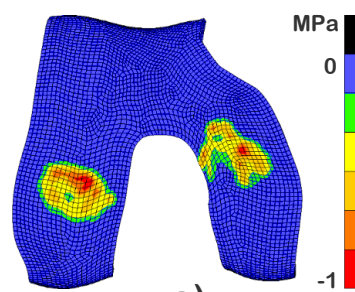

a)

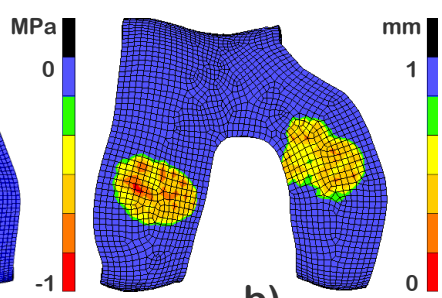

b)

Figure 7. Contact area in the knee, extension position. a) Contact pressure in MPa, b) Nodal distances in mm derived from MRI images

ion from $0^{\circ}$ to $63^{\circ}$ are depicted on the Figure 8 . It shows that as flexion increases the contact pressure tends to increase on the medial side of the tibial cartilage layer.

The simulation for the model with the menisci cannot be achieved above $30^{\circ}$ of knee flexion. The contact area and the contact pressure on the cartilage layers during the knee flexion from $0^{\circ}$ to $30^{\circ}$ are depicted on the Figure 9 It shows that the contact area are greater than without the menisci. 


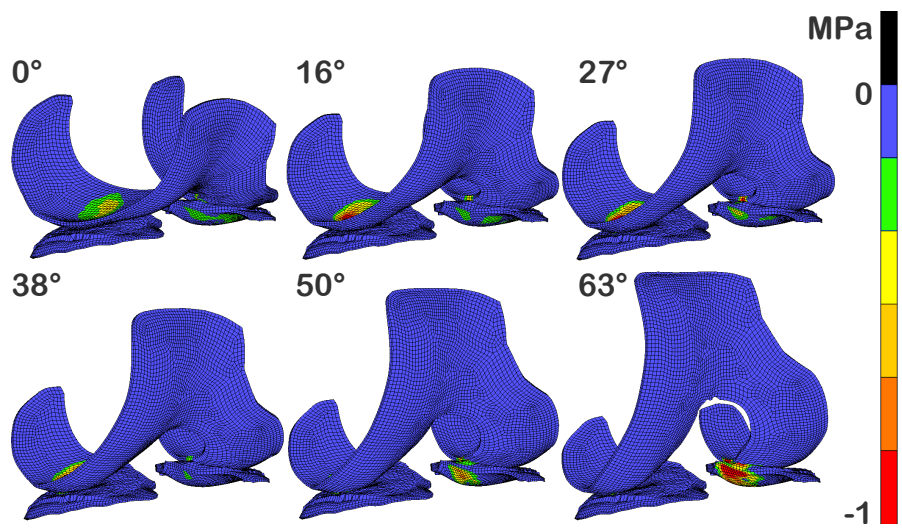

Figure 8. Contact pressure in $\mathrm{MPa}$ without the menisci from $0^{\circ}$ to $63^{\circ}$
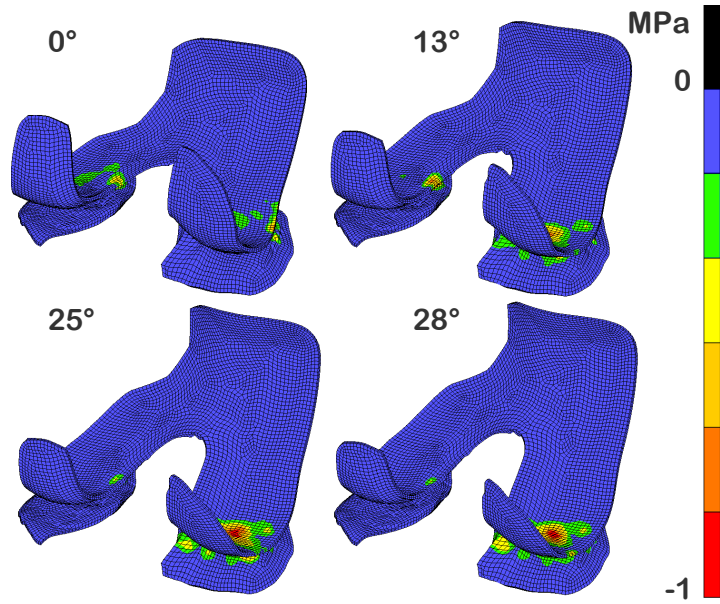

Figure 9. Contact pressure in $\mathrm{MPa}$ with the menisci from $0^{\circ}$ to $28^{\circ}$

\section{Conclusion and discussion}

This paper has presented a new methodology to build in vivo knee models able to compute the contact pressure distribution on the knee cartilage layers or menisci during a knee flexion-extension movement. The model presented include personalized kinematics boundary conditions and modeling of the bones, cartilage layers and menisci. This objective was achieved thanks to dedicated MRI protocols and modeling procedures. In particular the first part of the work has presented a new method to track the flexion-extension kinematics inside an MRI bore. The second part has presented a modeling procedure that allow both a good mesh generation on cartilage 
layers and menisci. This procedure provide also a mean to assess the reproducibility and accuracy of geometrical modeling, which matters when dealing with personalized finite element analysis. Eventually, these cartilage thickness maps computed from MRI images can also have clinical relevance.

Concerning this last aspect, the results show that rather than inter-operators accuracy the MRI sequence to be used is the main parameter to optimize since it makes the greatest variations.

The model was able to deduce contact area and contact pressure distribution on the cartilage layers for the full flexion-extension movement tracked by MRI. The more complicated model, with menisci between cartilage layers, cannot be processed for the full range of motion. This can be explained by the complexity of the model, 3 deformable structures in contact, and the ability of the finite element software chosen to handle this complexity.

At last, from an in vivo personalized modeling objective point of view, one major point to be discussed is that this model does not take into account the personalized material properties of the subject. There is nowadays no mean to deduce in vivo the material properties of either the cartilage layers nor the menisci. This aspect needs to be tackled in the future in order to improve the relevance of such models.

\section{References}

Chetverikov D., Stepanov D., Krsek P., “ Robust Euclidean alignment of 3D point sets: the Trimmed Iterative Closest Point algorithm”, Image and Vision Computing, vol. 23, $\mathrm{n}^{\circ} 3$, p. 299-309, 2005.

Donahue T., Hull M., Rashid M., Jacobs C., “ A finite element model of the human knee joint for the study of tibio-femoral contact", Journal of Biomechanical Engineering, vol. 124, p. 273-280, 2002. cartilage.

Donahue T., Hull M., Rashid M., Jacobs C., " How the stiffness of meniscal attachments and meniscal material properties affect tibio-femoral contact pressure computed using a validated finite element model of the human knee joint", Journal of Biomechanics, vol. 36, p. 19-34, 2003.

Ho Ba Tho M.-C., “ SIP 305 logiciel de pré-traitement d'images médicales, scanner, IRM”, 1993. copyright INSERM.

Ho Ba Tho M.-C., “ Bone and joints modelling with individualised geometric and mechanical properties derived from medical image", Computer Mechanics in Engineering and Sciences, vol. 3/4, p. 489-496, 2003.

Sangeux M., Marin F., Charleux F., Dürselen L., Tho M.-C. H. B., “ Quantification of the 3D relative movement of external marker sets vs. bones based on magnetic resonnace imaging", Clinical Biomechanics, vol. 21(9), p. 984-991, 2006.

Yao J., Snibbe J., Maloney M., Lerner A., “ Stresses and strains in the medial meniscus of an ACL deficient knee under anterior loading: A finite element analysis with image-based experimental validation”, Journal of Biomechanical Engineering, vol. 128, p. 135-141, 2006. 